\title{
Geographical Patterning of Physical Activity Prevalence in Iran: Spatial Analysis of 4 Pooled National Health Surveys Among 119,560 Adults
}

\author{
Mohammad Sahebkar, Hamid Heidarian Miri, Pardis Noormohammadpour, Amir Tiyuri, Reza Pakzad, \\ Nasrin Mansournia, Zahra Heidari, Mohammad Ali Mansournia, and Emmanuel Stamatakis
}

\begin{abstract}
Background: To investigate the geographical distribution of physical activity (PA) prevalence among adults aged 15-64 years old across Iran provinces using geographic maps. Methods: Data from 4 consecutive national surveys conducted between 2007 and 2010 were pooled to determine the geographical distribution. Prevalence of low PA with 95\% confidence interval was estimated by sociodemographic subpopulations over provinces using complex survey design. Results: In total, 119,560 participants (49.9\% females) were included in the analyses. The mean (SD) age of participants was 39.5 (14.3) years. The prevalence of the low PA in the pooled 2007-2010 was 35.8\% (95\% confidence interval, 34.1-37.6). The 3 provinces with the highest prevalence of low PA were Sistan and Baluchestan, Yazd, and Hormozgan. The results of hot spot analysis showed that the Kerman province was a hot spot, and Ilam, Kermanshah, Hamedan, and Markazi were cold spots for low PA. Ilam, Kohgiluyeh and Boyer-Ahmad, and Mazandaran had the highest total PA volume (metabolic equivalent minutes per week). Hot spot analysis showed that Ilam and Khuzestan provinces were hot spots for the total PA volume. Conclusions: The regions with low and high PA are predominately situated in the near center/southeast and west, respectively.
\end{abstract}

Keywords: epidemiology, national step survey, geographical pattern, exercise

Physical activity (PA) has well-established health benefits; low PA level is one of the most prevalent risk factors for noncommunicable disease worldwide. Regular PA has been shown to prevent cardiovascular diseases, type 2 diabetes, some cancers, hypertension, obesity, and depression; studies have also shown that low PA is considered a major risk factor for disability and is associated with significant economic burden in the world. ${ }^{1-3}$

According to a report by the general assembly of the World Health Organization in 2017, there has been little progress with reducing the burden of noncommunicable diseases. Reducing the prevalence of low PA requires a coordinated and long-term global action plan. ${ }^{4}$

The prevalence of low PA in the world is estimated to be $21.4 \%$, which varies from $2.6 \%$ to $63.3 \%$ according to wealth and

Sahebkar is with the Department of Social Medicine, School of Medicine, Sabzevar University of Medical Sciences, Sabzevar, Iran; and Student Research Committee, Sabzevar University of Medical Sciences, Sabzevar, Iran. Heidarian Miri is with the Social Determinants of Health Research Center, Mashhad University of Medical Sciences, Mashhad, Iran. Noormohammadpour and M.A. Mansournia are with Sports Medicine Research Center, Neuroscience Institute, Tehran University of Medical Sciences, Tehran, Iran. Tiyuri is with the Student Research Committee, Iran University of Medical Sciences, Tehran, Iran. Pakzad is with the Noor Research Center for Ophthalmic Epidemiology, Noor Eye Hospital, Tehran, Iran; and the Faculty of Health, Ilam University of Medical Sciences, Ilam, Iran. N. Mansournia is with the Department of Endocrinology and Metabolism, AJA University of Medical Sciences, Tehran, Iran. M.A. Mansournia is with the Department of Epidemiology and Biostatistics, School of Public Health, Tehran University of Medical Sciences, Tehran, Iran. Heidari is with the Department of Endocrinology and Metabolism, Zahedan University of Medical Sciences, Zahedan, Iran. Stamatakis is with Charles Perkins Centre, Epidemiology Unit, The University of Sydney, Sydney, NSW, Australia; and Prevention Research Collaboration, School of Public Health, The University of Sydney, Sydney, NSW, Australia. M.A. Mansournia (mansournia_ ma@yahoo.com) is corresponding author. development status across countries. According to the latest national study on risk factors of noncommunicable diseases in Iran in 2011 , the prevalence of physical inactivity among adults increased from $35 \%$ in 2007 to $39.1 \% .^{5,6}$ In the systematic review carried out by Fakhrzadeh et al, ${ }^{7}$ the prevalence of low PA in Iran is high and ranges from $30 \%$ to $70 \%$ in different studies.

Low PA has been identified as the fourth cause of global death (6\% of deaths globally). Furthermore, low PA has been shown to contribute to $21 \%-25 \%$ of breast and colon cancers, $27 \%$ of type 2 diabetes, and roughly $30 \%$ of ischemic heart disease burden. ${ }^{8}$

Understanding the geographical distribution of PA can help prioritize distribution of health resources within countries and to potentially improve their effectiveness. ${ }^{9,10}$

Spatial analysis is used to examine distribution patterns of a health outcome, behavior, or population characteristic. Such analysis can reveal if differences among regions are random or occur at a consistent geographical pattern. Also, if such patterns exist, they will be detected with quantitative measures. ${ }^{1,12}$ Examining PA patterns using geographic information systems (GIS) can inform national PA policy by identifying target areas with low PA. ${ }^{13,14}$

To the best of our knowledge, no spatial analysis of PA patterns of the Iranian population has been published. The aim of this population-based study was to investigate the distribution of PA prevalence across different provinces of Iran using geographic maps.

\section{Methods}

\section{The Study Area}

Iran, a country in the Middle East with the extent of $1,648,195 \mathrm{~km}^{2}$, has been ranked as the 17th largest country in the world with a population of over 75 million (2011). It possesses a number of 
unique environmental and topological features, such as vast length and breadth, topographic diversity, and large variation in altitude including a peak of $5671 \mathrm{~m}$ above sea level. Iran has been currently divided into 31 provinces. ${ }^{15}$

\section{Data Source}

In this cross-sectional population-based study, we merged the data obtained from 4 consecutive rounds of national surveys, which were carried out from 2007 to 2010 in Iran. Approximately 30,000 noninstitutionalized Iranian people aged 15-64 years were recruited in each survey. Respondents were 119,560 individuals between 15 and 64 years old who had valid PA data. Participants were sampled using stratified cluster random sampling techniques, with the provinces were defined to be as strata. Random samples of 1000 individuals were selected from each stratum. The primary sampling units were assumed as blocks of buildings in both residential areas (rural and urban) and were chosen randomly from the list of postal codes provided by Iran's postal service (10 digits), based on a systematic sampling method. A cluster consisted of 20 individuals, 2 males and 2 females in each age group (ie, 15-24, 25-34, 35-44, 45-54, and 55-64 y), and assessors interviewed households in each block until the clusters were completed. The detailed methodology of the survey sampling has been described previously. ${ }^{16}$ This study was approved by institutional review board of Tehran University of Medical Sciences with ethics committee code: IR.TUMS.SPH.REC.1395.514.

\section{Physical Activity}

Self-reported PA was measured using the Farsi (or Persian) version $^{17,18}$ of the Global PA Questionnaire (GPAQ, version 2) ${ }^{19}$ developed by World Health Organization. GPAQ has demonstrated acceptable validity and reliability for evaluating PA in a national surveillance medium-income country. The questionnaire includes 16 questions to assess the PA, in moderate and vigorous intensity, in 3 domains: during working, commuting, and leisure times. ${ }^{20}$ The GPAQ assessed participation in moderate-intensity and vigorousintensity activities, and time spent in sedentary behavior (watching television, sitting and, resting) on an ordinary day. Energy expenditure was calculated using metabolic equivalents (METs) values of each reported activity. ${ }^{21}$ Moderate-intensity and vigorous-intensity activities were assigned 4 METs and 8 METs, respectively. ${ }^{19}$ Total PA (TPA; MET minutes per week) was considered as the sum of all METs multiplied by work, transportation, and recreation times per week. $^{22}$ Further details on GPAQ can be found in other published sources. ${ }^{19,20}$

According to GPAQ framework, ${ }^{19}$ the PA levels were classified as follows:

"High level: Vigorous intensity activity in 3 days with a minimum total expenditure of $1500 \mathrm{MET}-\mathrm{min} /$ week; or a mixture of activities, such as walking and moderate/vigorous-intensity activities in at least 7 days with at least consumption of 3000 MET-min/week.

Moderate level: Persons who did not match the criteria for the high level and who reported vigorous intensity activity for at least 20 minutes in 3 or more days per week; or moderate intensity activity or walking for at least 30 minutes on 5 or more days per week; or any mixture of vigorous or moderate intensity activity or walking with minimum $600 \mathrm{MET}$-min/week.

Low level: Persons not classified in any of the previously mentioned groups come at this level."

\section{Statistical Analysis}

Questionnaire data were collected according to the World Health Organization STEPwise guideline for health surveillance studies. $^{22}$ The gathered data were analyzed on August 2018. The multistage sampling design was taken into account using standard complex survey analysis method We applied inverse probability weighting and linearized (robust) standard error to adjust for unequal probability of selection and clustering, respectively (but not frequency weighting), which does not increase the sample size. ${ }^{23}$ We determined the final weight by multiplying the subsequent weights: First, sampling weight that is the inverse probability of each subject sampling and second, nonresponse weight that is the inverse probability of response in each sexage group.

Continuous variables were summarized as median (interquartile range), and categorical variables were presented as number (percentage). The overall prevalence of each level of PA (low, moderate, and high) and mean of METs with $95 \%$ confidence intervals were reported. All the analyses were performed using Stata (version 12; Stata Corp, College Station, TX), and all maps were generated using ArcGIS Desktop (version 10.1; ESRI, Redlands, CA).

\section{Hot Spot Analysis}

For hot spot analysis, the most recently updated electronic map of Iran was linked to the Excel file that contained prevalence of PA for Iran's provinces. Then, to assess the presence of spatial-clustering, Getis-Ord $\mathrm{Gi}^{*}$ statistic score was calculated for each province based on Equation 1 and was compared with its corresponding expected value. Then, a province is considered hot/cold spot if its $\mathrm{G}$ score is greater than or less than its expected value. Indeed, this difference needs to be more than to be justified by random error to be statistically significant. ${ }^{16,24-26}$ This means that if the province has a difference of its expected difference of more than +1.96 and -1.96 , then the province is considered a significant hot/cold spot, and if the difference is between -1.96 and +1.96 , then the province is considered nonsignificant.

The expected values are defined based on Fixed Distance Band method. According to this method, the expected value of a province is calculated as the weighted average of the observed values of its neighboring provinces within a specified critical distance range in a way that nearer provinces receive a higher weight. For determination of critical distance, we used the Euclidean distance method; that is, the straight and minimum distance between 2 polygons center. Center of polygons is determined by the centroid method. ${ }^{26}$

$$
G_{i} \cdot \text { Statistics }=\frac{\sum_{j=1}^{n} W_{i} X_{j}-X \sum_{j=1}^{n} W_{i j}}{S \sqrt{\left[\frac{n \sum_{j=1}^{n} W_{i \cdot j}^{2}-\left(\sum_{j=1}^{n} W_{i, j}\right)}{n-1}\right]}} .
$$

Equation 1: Getis-Ord Gi* (Gi* Statistics), where $X_{j}$ is the incidence of PA for province $j, W_{i \cdot j}$ is the spatial weight between province $i$ and $j$, and $n$ is the total number of provinces.

\section{Results}

The study sample comprised 119,560 individuals; $50.1 \%$ were males and $49.9 \%$ were females. The response rate was $90 \%$ for each survey. The mean (SD) age of participants was $39.5(0.04)$ 
Table 1 The Characteristic of the Population Under Study From 2007 to 2010 (Crude/Unweighted Percentages), $\mathrm{N}=1119,560$

\begin{tabular}{lc}
\hline Variables & $\mathbf{n}(\%)$ \\
\hline Age group, y & \\
$15-24$ & $24,188(20.23)$ \\
$25-34$ & $23,930(20.02)$ \\
$35-44$ & $23,958(20.04)$ \\
$45-54$ & $24,028(20.10)$ \\
$55-64$ & $23,456(19.62)$ \\
Sex & \\
Male & $59,794(50.01)$ \\
Female & $59,766(49.99)$ \\
Residential area & \\
Urban & $70,971(59.36)$ \\
Rural & $48,586(40.64)$ \\
\hline
\end{tabular}

Note: Study participants with missing data were excluded. years, and $59.3 \%$ of the participants were from urban areas. The demographic data of the study population are provided in Table 1.

The prevalence estimate of the low PA in the whole population from 2007 to 2010 was estimated to be $35.8 \%$ (95\% confidence interval, 34.1-37.6). The results of hot spot analysis showed that Kerman province was a low PA hot region $(P<.05)$. Ilam, Kermanshah, Hamedan, and Markazi were low PA cold spots $(P<.05)$ (Figure 1A). Razavi Khorasan $(P<.01)$, Semnan, Northern Khorasan, and Golestan provinces $(P<.05)$ were hot spots for moderate PA level (Figure 1B). Ilam $(P<.01)$, and Kermanshah, Hamedan, Markazi, and Kurdistan provinces $(P<.05)$ were hot spots for high PA level. Fars province was a cold spot for high PA level $(P<.05)$ (Figure 1C). Table 2 presents the levels of PA by gender in Iranian adults aged 15-64 years from 2007 to 2010.

Hot spot analysis showed that in men, Ilam and Khuzestan provinces were hot spots for the TPA volume (MET minutes per week) $(P<.05)$. Although the provinces of Kermanshah and Lorestan were hot regions for TPA volume (MET minutes per week), the differences were not statistically significant (Figure 1D).
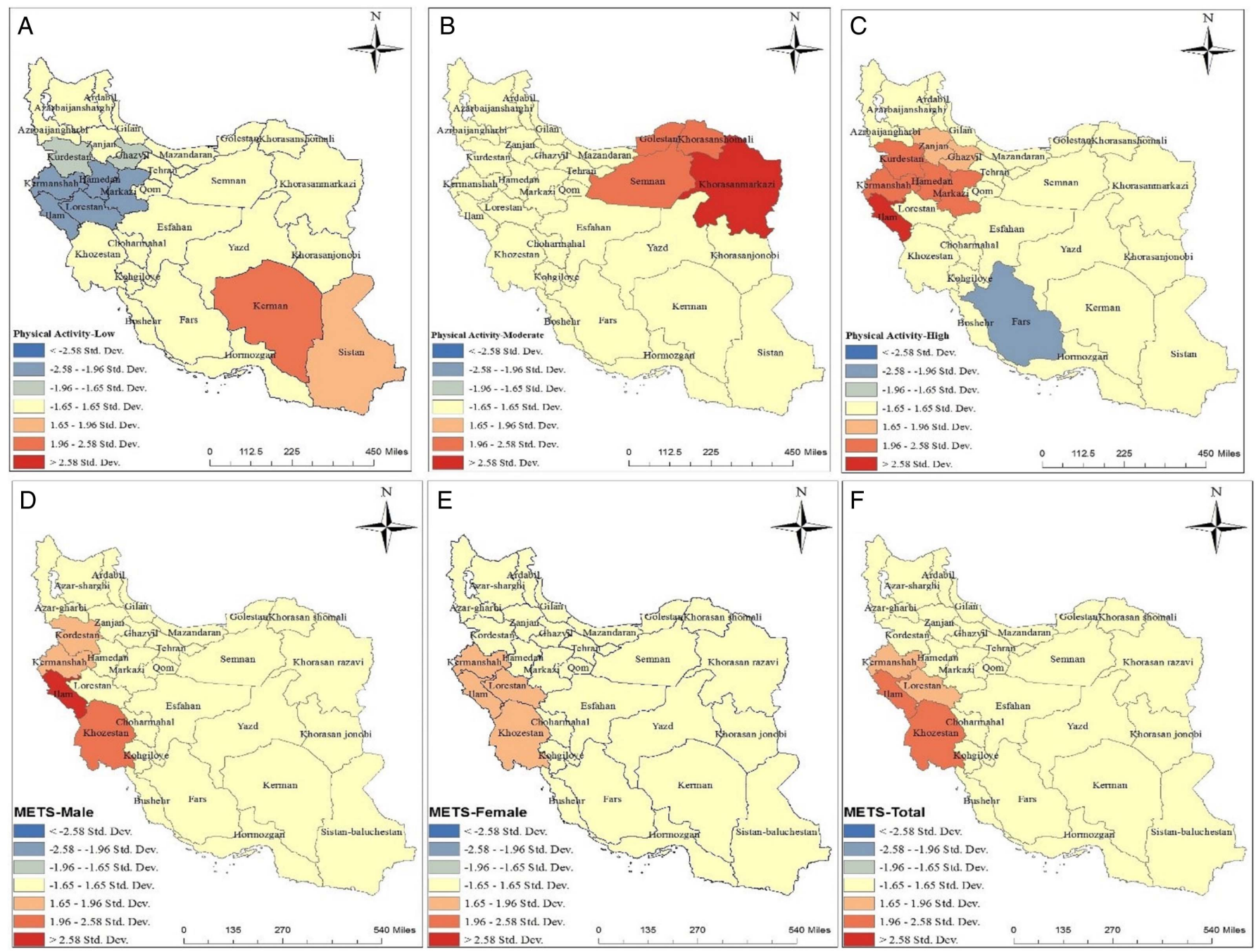

Figure 1 - Low level of physical activity (A), moderate level of physical activity (B), high level of physical activity (C), hot spot maps for METs in males (D), METs in females (E), and METs in total (F). METs indicate metabolic equivalents. 
Table 2 The Levels of Physical Activity by Gender in Iranian Adults Aged 15-64 years, 2007-2010

\begin{tabular}{|c|c|c|c|c|c|c|c|c|c|}
\hline \multirow[b]{3}{*}{ Province } & \multicolumn{9}{|c|}{ Levels of physical activity } \\
\hline & \multicolumn{3}{|c|}{$\begin{array}{l}\text { Low } \%(n) \\
95 \% \text { confidence interval }\end{array}$} & \multicolumn{3}{|c|}{$\begin{array}{l}\text { Moderate } \%(n) \\
95 \% \text { confidence interval }\end{array}$} & \multicolumn{3}{|c|}{$\begin{array}{c}\text { High \% }(n) \\
95 \% \text { confidence interval }\end{array}$} \\
\hline & Male & Female & Total & Male & Female & Total & Male & Female & Total \\
\hline East Azerbaijan & $\begin{array}{l}24.5(525) \\
22.6-26.4\end{array}$ & $\begin{array}{l}47.9(972) \\
41.2-54.7\end{array}$ & $\begin{array}{c}36.1(1497) \\
32.5-39.8\end{array}$ & $\begin{array}{l}20.1(407) \\
16.9-23.5\end{array}$ & $\begin{array}{l}25.7(493) \\
19.8-32.7\end{array}$ & $\begin{array}{l}22.9(900) \\
18.6-27.7\end{array}$ & $\begin{array}{c}55.4(1053) \\
53.9-56.8\end{array}$ & $\begin{array}{l}26.2(534) \\
21.2-31.9\end{array}$ & $\begin{array}{l}41(1587) \\
37.9-44.1\end{array}$ \\
\hline West Azerbaijan & $\begin{array}{l}23.6(517) \\
21.1-26.3\end{array}$ & $\begin{array}{c}53.4(1052) \\
50.1-56.7\end{array}$ & $\begin{array}{c}38.3(1569) \\
35.5-41.1\end{array}$ & $\begin{array}{l}22.2(457) \\
20.1-24.5\end{array}$ & $\begin{array}{c}23.24(464) \\
21.6-24.9\end{array}$ & $\begin{array}{l}22.7(921) \\
22.1-23.3\end{array}$ & $\begin{array}{c}54.1(1025) \\
51.2-56.9\end{array}$ & $\begin{array}{l}23.3(486) \\
20.9-25.8\end{array}$ & $\begin{array}{l}38.9(1511) \\
36.4-41.4\end{array}$ \\
\hline Ardabil & $\begin{array}{l}28.5(618) \\
23.9-33.6\end{array}$ & $\begin{array}{c}49.4(1003) \\
40.7-58.1\end{array}$ & $\begin{array}{c}38.8(1621) \\
32.4-45.7\end{array}$ & $\begin{array}{l}17.7(380) \\
14.6-21.4\end{array}$ & $\begin{array}{l}21.5(420) \\
18.5-24.9\end{array}$ & $\begin{array}{l}19.6(800) \\
17.1-22.4\end{array}$ & $\begin{array}{l}53.6(999) \\
46.4-60.7\end{array}$ & $\begin{array}{l}28.9(572) \\
21.1-38.3\end{array}$ & $\begin{array}{c}41.4(1571) \\
34.1-49.3\end{array}$ \\
\hline Esfahan & $\begin{array}{l}26.5(582) \\
24.5-28.6\end{array}$ & $\begin{array}{c}50.4(1017) \\
44.8-56.1\end{array}$ & $\begin{array}{c}38.3(1599) \\
34.9-41.8\end{array}$ & $\begin{array}{l}24.3(492) \\
18.6-31.1\end{array}$ & $\begin{array}{l}31.9(633) \\
25.8-38.7\end{array}$ & $\begin{array}{c}28.1(1125) \\
22.3-34.6\end{array}$ & $\begin{array}{l}49.1(923) \\
44.5-53.7\end{array}$ & $\begin{array}{l}17.6(349) \\
15.4-20.1\end{array}$ & $\begin{array}{c}33.5(1272) \\
30.3-37.1\end{array}$ \\
\hline Ilam & $\begin{array}{l}18.1(395) \\
14.7-22.0\end{array}$ & $\begin{array}{c}36.08(688) \\
30.2-42.3\end{array}$ & $\begin{array}{c}27.1(1083) \\
23.7-30.4\end{array}$ & $\begin{array}{l}18.1(387) \\
13.6-23.8\end{array}$ & $\begin{array}{c}23.1(463) \\
19.6-26.9\end{array}$ & $\begin{array}{r}20.6(850) \\
17.2-24.7\end{array}$ & $\begin{array}{c}63.6(1212) \\
55.1-71.4\end{array}$ & $\begin{array}{l}40.8(848) \\
32.1-50.2\end{array}$ & $\begin{array}{c}52.3(2060) \\
45.8-58.8\end{array}$ \\
\hline Bushehr & $\begin{array}{l}33.8(767) \\
30.3-37.6\end{array}$ & $\begin{array}{c}58.8(1175) \\
54.3-63.1\end{array}$ & $\begin{array}{c}46.1(1942) \\
42.7-49.6\end{array}$ & $\begin{array}{l}18.7(377) \\
17.1-20.4\end{array}$ & $\begin{array}{l}26.1(499) \\
25.2-26.7\end{array}$ & $\begin{array}{c}22.32(876) \\
21.5-23.1\end{array}$ & $\begin{array}{c}47.43(855) \\
43.5-51.3\end{array}$ & $\begin{array}{l}15.1(324) \\
10.8-20.7\end{array}$ & $\begin{array}{c}31.4(1179) \\
28.1-35.1\end{array}$ \\
\hline Tehran & $\begin{array}{l}27.7(626) \\
23.4-32.4\end{array}$ & $\begin{array}{c}43.4(890) \\
37.4-49.7\end{array}$ & $\begin{array}{c}35.5(1516) \\
30.5-40.8\end{array}$ & $\begin{array}{l}25.2(528) \\
24.1-26.4\end{array}$ & $\begin{array}{l}35.5(696) \\
33.1-37.9\end{array}$ & $\begin{array}{c}30.3(1224) \\
28.7-31.9\end{array}$ & $\begin{array}{l}47.1(841) \\
42.2-51.8\end{array}$ & $\begin{array}{l}21.1(410) \\
16.4-26.5\end{array}$ & $\begin{array}{c}34.1(1251) \\
29.4-39.1\end{array}$ \\
\hline Chaharmahal and Bakhtiari & $\begin{array}{l}19.9(446) \\
12.9-29.4\end{array}$ & $\begin{array}{l}40.4(821) \\
33.1-48.1\end{array}$ & $\begin{array}{c}30.1(1267) \\
23.1-38.2\end{array}$ & $\begin{array}{l}14.6(299) \\
11.5-18.4\end{array}$ & $\begin{array}{l}25.1(460) \\
22.8-27.4\end{array}$ & $\begin{array}{l}19.7(759) \\
17.2-22.5\end{array}$ & $\begin{array}{c}65.4(1273) \\
53.9-75.3\end{array}$ & $\begin{array}{l}34.5(689) \\
25.5-44.7\end{array}$ & $\begin{array}{c}50.1(1962) \\
40.4-59.8\end{array}$ \\
\hline Razavi Khorasan & $\begin{array}{l}21.2(479) \\
19.3-23.3\end{array}$ & $\begin{array}{l}37.9(763) \\
33.4-42.6\end{array}$ & $\begin{array}{c}29.5(1242) \\
26.3-32.8\end{array}$ & $\begin{array}{l}21.7(453) \\
20.7-22.7\end{array}$ & $\begin{array}{l}30.9(586) \\
26.5-35.6\end{array}$ & $\begin{array}{c}26.2(1039) \\
24.1-28.6\end{array}$ & $\begin{array}{c}57.1(1062) \\
54.1-59.9\end{array}$ & $\begin{array}{l}31.1(638) \\
24.6-38.2\end{array}$ & $\begin{array}{c}44.2(1700) \\
39.7-48.8\end{array}$ \\
\hline Khuzestan & $\begin{array}{l}26.1(601) \\
23.4-28.8\end{array}$ & $\begin{array}{c}55.1(1110) \\
49.8-60.1\end{array}$ & $\begin{array}{c}40.3(1711) \\
36.5-44.2\end{array}$ & $\begin{array}{c}20.5(419) \\
18.5-22.8\end{array}$ & $\begin{array}{l}25.5(487) \\
23.6-27.5\end{array}$ & $\begin{array}{l}23.1(906) \\
22.4-23.6\end{array}$ & $\begin{array}{l}53.3(941) \\
48.9-57.7\end{array}$ & $\begin{array}{l}19.4(373) \\
16.1-23.2\end{array}$ & $\begin{array}{c}36.6(1314) \\
32.8-40.5\end{array}$ \\
\hline Zanjan & $\begin{array}{l}19.5(440) \\
14.1-26.5\end{array}$ & $\begin{array}{c}41(811) \\
36.2-45.9\end{array}$ & $\begin{array}{c}30.1(1251) \\
25.1-35.6\end{array}$ & $\begin{array}{c}20.3(410) \\
17.8-23.1\end{array}$ & $\begin{array}{l}29.8(579) \\
28.1-31.6\end{array}$ & $\begin{array}{l}25.1(989) \\
23.8-26.2\end{array}$ & $\begin{array}{c}60.1(1151) \\
51.9-67.8\end{array}$ & $\begin{array}{l}29.1(596) \\
24.1-34.6\end{array}$ & $\begin{array}{c}44.8(1747) \\
38.5-51.3\end{array}$ \\
\hline Semnan & $\begin{array}{l}31.2(671) \\
26.7-36.1\end{array}$ & $\begin{array}{l}48.9(974) \\
43.9-53.8\end{array}$ & $\begin{array}{c}39.9(1645) \\
35.3-44.7\end{array}$ & $\begin{array}{l}18.3(407) \\
16.9-19.7\end{array}$ & $\begin{array}{l}33.3(656) \\
31.3-35.4\end{array}$ & $\begin{array}{c}25.7(1063) \\
25.2-26.2\end{array}$ & $\begin{array}{l}50.4(917) \\
45.5-55.4\end{array}$ & $\begin{array}{l}17.7(396) \\
13.9-22.2\end{array}$ & $\begin{array}{c}34.3(1286) \\
29.9-38.9\end{array}$ \\
\hline Sistan and Baluchestan & $\begin{array}{l}34.5(742) \\
30.9-38.2\end{array}$ & $\begin{array}{c}61.7(1210) \\
57.1-66.1\end{array}$ & $\begin{array}{c}47.9(1952) \\
44.1-51.8\end{array}$ & $\begin{array}{l}21.2(435) \\
17.3-25.7\end{array}$ & $\begin{array}{l}20.1(413) \\
17.1-23.5\end{array}$ & $\begin{array}{l}20.6(848) \\
17.5-24.1\end{array}$ & $\begin{array}{l}44.2(785) \\
36.9-51.8\end{array}$ & $\begin{array}{l}18.1(346) \\
13.3-24.1\end{array}$ & $\begin{array}{c}31.3(1131) \\
25.2-38.1\end{array}$ \\
\hline Fars & $\begin{array}{l}32.5(697) \\
24.6-41.5\end{array}$ & $\begin{array}{l}51.1(978) \\
38.3-63.8\end{array}$ & $\begin{array}{c}41.7(1675) \\
31.7-52.3\end{array}$ & $\begin{array}{l}23.3(465) \\
20.4-26.4\end{array}$ & $\begin{array}{l}25.4(493) \\
23.2-27.2\end{array}$ & $\begin{array}{l}24.3(958) \\
22.8-25.9\end{array}$ & $\begin{array}{l}44.1(805) \\
33.1-55.7\end{array}$ & $\begin{array}{l}23.4(478) \\
13.4-37.6\end{array}$ & $\begin{array}{c}33.9(1283) \\
23.3-46.3\end{array}$ \\
\hline Qazvin & $\begin{array}{l}29.1(635) \\
24.4-34.2\end{array}$ & $\begin{array}{c}49.3(979) \\
45.9-52.7\end{array}$ & $\begin{array}{c}39.1(1614) \\
35.1-43.2\end{array}$ & $\begin{array}{c}20.1(430) \\
18.3-21.8\end{array}$ & $\begin{array}{l}26.9(543) \\
24.1-29.9\end{array}$ & $\begin{array}{l}23.4(973) \\
21.2-25.7\end{array}$ & $\begin{array}{l}50.8(937) \\
46.8-54.8\end{array}$ & $\begin{array}{l}23.7(472) \\
21.7-25.8\end{array}$ & $\begin{array}{c}37.4(1409) \\
34.8-40.1\end{array}$ \\
\hline Qom & $\begin{array}{l}29.3(655) \\
25.5-33.4\end{array}$ & $\begin{array}{c}54.7(1123) \\
46.6-62.6\end{array}$ & $\begin{array}{c}41.8(1778) \\
36.1-47.9\end{array}$ & $\begin{array}{l}27.7(545) \\
24.2-31.5\end{array}$ & $\begin{array}{l}29.3(566) \\
25.7-33.2\end{array}$ & $\begin{array}{c}28.5(1111) \\
26.5-30.6\end{array}$ & $\begin{array}{l}42.9(794) \\
37.2-48.7\end{array}$ & $\begin{array}{l}15.8(312) \\
11.8-21.1\end{array}$ & $\begin{array}{c}29.5(1106) \\
24.9-34.6\end{array}$ \\
\hline Kurdistan & $\begin{array}{l}22.1(469) \\
18.4-26.2\end{array}$ & $\begin{array}{l}44.1(853) \\
36.1-52.2\end{array}$ & $\begin{array}{c}32.9(1322) \\
27.4-38.9\end{array}$ & $\begin{array}{c}20.1(392) \\
18.4-21.6\end{array}$ & $\begin{array}{l}21.4(417) \\
20.1-22.8\end{array}$ & $\begin{array}{c}20.7(809) \\
19.7-21.7\end{array}$ & $\begin{array}{c}57.9(1134) \\
54.1-61.6\end{array}$ & $\begin{array}{l}34.4(727) \\
27.6-41.9\end{array}$ & $\begin{array}{c}46.3(1861) \\
41.3-51.4\end{array}$ \\
\hline Kerman & $\begin{array}{l}26.3(586) \\
23.6-29.2\end{array}$ & $\begin{array}{c}54.6(1099) \\
50.3-58.7\end{array}$ & $\begin{array}{c}40.3(1685) \\
38.1-42.5\end{array}$ & $\begin{array}{l}18.8(390) \\
15.6-22.4\end{array}$ & $\begin{array}{l}22.6(425) \\
20.8-24.4\end{array}$ & $\begin{array}{l}20.6(815) \\
19.6-21.7\end{array}$ & $\begin{array}{c}54.8(1019) \\
51.8-57.9\end{array}$ & $\begin{array}{l}22.7(469) \\
20.2-25.5\end{array}$ & $\begin{array}{c}39.1(1488) \\
36.6-41.4\end{array}$ \\
\hline Kermanshah & $\begin{array}{l}20.2(474) \\
17.1-23.7\end{array}$ & $\begin{array}{l}43.2(831) \\
32.9-54.2\end{array}$ & $\begin{array}{c}31.6(1305) \\
25.5-38.4\end{array}$ & $\begin{array}{l}24.7(518) \\
22.3-27.3\end{array}$ & $\begin{array}{l}32.1(664) \\
29.9-34.3\end{array}$ & $\begin{array}{c}28.3(1182) \\
26.2-30.4\end{array}$ & $\begin{array}{c}55.1(1006) \\
50.4-59.5\end{array}$ & $\begin{array}{c}24.7(504) \\
14.8-38.1\end{array}$ & $\begin{array}{c}40.1(1510) \\
32.4-48.1\end{array}$ \\
\hline Kohgiluyeh and Boyer-Ahmad & $\begin{array}{l}15.1(349) \\
11.9-18.8\end{array}$ & $\begin{array}{l}29.7(625) \\
23.4-36.8\end{array}$ & $\begin{array}{l}22.3(974) \\
17.6-27.7\end{array}$ & $\begin{array}{c}21.6(448) \\
17.7-26.1\end{array}$ & $\begin{array}{l}28.6(548) \\
21.9-36.3\end{array}$ & $\begin{array}{l}25.1(996) \\
20.6-30.2\end{array}$ & $\begin{array}{c}63.2(1198) \\
57.6-68.5\end{array}$ & $\begin{array}{l}41.6(826) \\
36.8-46.5\end{array}$ & $\begin{array}{c}52.5(2024) \\
48.3-56.7\end{array}$ \\
\hline Golestan & $\begin{array}{l}25.7(589) \\
22.6-29.1\end{array}$ & $\begin{array}{c}52.3(1061) \\
48.1-56.5\end{array}$ & $\begin{array}{c}38.8(1650) \\
35.6-42.2\end{array}$ & $\begin{array}{l}17.2(379) \\
15.7-18.9\end{array}$ & $\begin{array}{l}23.8(450) \\
22.5-25.2\end{array}$ & $\begin{array}{c}20.5(829) \\
19.3-21.7\end{array}$ & $\begin{array}{c}56.9(1029) \\
53.7-60.1\end{array}$ & $\begin{array}{l}23.8(475) \\
20.4-27.5\end{array}$ & $\begin{array}{c}40.6(1504) \\
38.1-43.1\end{array}$ \\
\hline Gilan & $\begin{array}{c}20.6(441) \\
15.7-26.6\end{array}$ & $\begin{array}{l}44.6(865) \\
38.1-51.3\end{array}$ & $\begin{array}{c}32.4(1306) \\
26.7-38.7\end{array}$ & $\begin{array}{l}19.5(395) \\
16.7-22.7\end{array}$ & $\begin{array}{l}27.1(532) \\
26.2-27.8\end{array}$ & $\begin{array}{l}23.2(927) \\
21.6-24.9\end{array}$ & $\begin{array}{c}59.8(1147) \\
51.8-67.2\end{array}$ & $\begin{array}{l}28.3(593) \\
22.4-35.1\end{array}$ & $\begin{array}{c}44.2(1740) \\
37.3-51.3\end{array}$ \\
\hline Lurestan & $\begin{array}{l}13.2(295) \\
12.3-14.1\end{array}$ & $\begin{array}{c}34.49(678) \\
28.4-41.1\end{array}$ & $\begin{array}{c}23.74(972) \\
20.4-27.3\end{array}$ & $\begin{array}{c}16.06(345) \\
13.7-18.6\end{array}$ & $\begin{array}{l}33.3(628) \\
31.4-35.3\end{array}$ & $\begin{array}{l}24.6(973) \\
23.2-26.1\end{array}$ & $\begin{array}{c}70.7(1353) \\
68.6-72.7\end{array}$ & $\begin{array}{l}32.1(682) \\
26.6-38.7\end{array}$ & $\begin{array}{c}51.6(2035) \\
48.7-54.5\end{array}$ \\
\hline Mazandaran & $\begin{array}{l}15.5(351) \\
13.8-17.4\end{array}$ & $\begin{array}{l}31.1(625) \\
28.8-33.4\end{array}$ & $\begin{array}{l}23.2(976) \\
21.2-24.9\end{array}$ & $\begin{array}{l}19.4(400) \\
16.1-23.2\end{array}$ & $\begin{array}{l}29.9(565) \\
27.5-32.5\end{array}$ & $\begin{array}{l}24.6(965) \\
21.8-27.6\end{array}$ & $\begin{array}{c}65.1(1250) \\
62.1-67.8\end{array}$ & $\begin{array}{l}38.8(804) \\
35.8-41.9\end{array}$ & $\begin{array}{c}52.1(2054) \\
49.2-55.1\end{array}$ \\
\hline Markazi & $\begin{array}{l}14.8(342) \\
12.7-17.3\end{array}$ & $\begin{array}{l}45.1(901) \\
34.2-56.5\end{array}$ & $\begin{array}{c}29.8(1243) \\
24.1-36.1\end{array}$ & $\begin{array}{l}20.8(447) \\
18.2-23.6\end{array}$ & $\begin{array}{l}29.2(578) \\
23.9-35.1\end{array}$ & $\begin{array}{c}24.9(1025) \\
21.1-29.2\end{array}$ & $\begin{array}{c}64.3(1210) \\
59.7-68.6\end{array}$ & $\begin{array}{l}25.6(519) \\
18.1-35.1\end{array}$ & $\begin{array}{c}45.2(1729) \\
40.1-50.5\end{array}$ \\
\hline Hormozgan & $\begin{array}{l}34.5(756) \\
29.4-40.1\end{array}$ & $\begin{array}{c}58.5(1172) \\
50.3-66.2\end{array}$ & $\begin{array}{c}46.4(1937) \\
39.9-53.1\end{array}$ & $\begin{array}{l}22.6(470) \\
20.4-25.1\end{array}$ & $\begin{array}{l}24.7(497) \\
21.2-28.6\end{array}$ & $\begin{array}{l}23.6(967) \\
22.4-24.9\end{array}$ & $\begin{array}{l}42.7(749) \\
37.1-48.7\end{array}$ & $\begin{array}{l}16.7(341) \\
12.1-22.7\end{array}$ & $\begin{array}{c}29.9(1090) \\
24.8-35.6\end{array}$ \\
\hline
\end{tabular}


Table 2 (continued)

\begin{tabular}{|c|c|c|c|c|c|c|c|c|c|}
\hline \multirow[b]{3}{*}{ Province } & \multicolumn{9}{|c|}{ Levels of physical activity } \\
\hline & \multicolumn{3}{|c|}{$\begin{array}{c}\text { Low \% }(n) \\
95 \% \text { confidence interval }\end{array}$} & \multicolumn{3}{|c|}{$\begin{array}{l}\text { Moderate } \%(n) \\
95 \% \text { confidence interval }\end{array}$} & \multicolumn{3}{|c|}{$\begin{array}{l}\text { High } \%(n) \\
95 \% \text { confidence interval }\end{array}$} \\
\hline & Male & Female & Total & Male & Female & Total & Male & Female & Total \\
\hline Hamedan & $\begin{array}{l}18.7(420) \\
14.9-23.3\end{array}$ & $\begin{array}{l}47.8(938) \\
45.1-50.5\end{array}$ & $\begin{array}{c}33.1(1358) \\
29.9-36.4\end{array}$ & $\begin{array}{l}22.4(461) \\
18.8-26.6\end{array}$ & $\begin{array}{l}31.6(641) \\
30.3-32.9\end{array}$ & $\begin{array}{l}27(1102) \\
24.7-29.3\end{array}$ & $\begin{array}{c}58.7(1117) \\
51.1-66.1\end{array}$ & $\begin{array}{c}20.5(420) \\
18.1-23.2\end{array}$ & $\begin{array}{c}39.8(1537) \\
35.2-44.7\end{array}$ \\
\hline Yazd & $\begin{array}{l}38.1(784) \\
33.2-43.1\end{array}$ & $\begin{array}{c}57.5(1151) \\
53.5-61.4\end{array}$ & $\begin{array}{c}47.6(1935) \\
43.6-51.7\end{array}$ & $\begin{array}{l}16.9(351) \\
14.9-19.1\end{array}$ & $\begin{array}{l}26.2(516) \\
23.5-29.2\end{array}$ & $\begin{array}{l}21.5(867) \\
19.7-23.4\end{array}$ & $\begin{array}{l}45.1(865) \\
41.3-48.8\end{array}$ & $\begin{array}{l}16.1(331) \\
13.5-19.2\end{array}$ & $\begin{array}{c}30.8(1196) \\
27.6-34.2\end{array}$ \\
\hline South Khorasan & $\begin{array}{l}22.3(457) \\
18.5-26.7\end{array}$ & $\begin{array}{l}36.4(723) \\
31.8-41.4\end{array}$ & $\begin{array}{c}29.3(1180) \\
27.2-31.5\end{array}$ & $\begin{array}{l}20.4(428) \\
19.1-21.8\end{array}$ & $\begin{array}{l}29.2(551) \\
26.2-32.4\end{array}$ & $\begin{array}{l}24.7(979) \\
22.7-26.9\end{array}$ & $\begin{array}{c}57.1(1113) \\
52.9-61.3\end{array}$ & $\begin{array}{l}34.2(724) \\
26.9-42.4\end{array}$ & $\begin{array}{c}45.8(1837) \\
42.2-49.5\end{array}$ \\
\hline North Khorasan & $\begin{array}{l}24.1(516) \\
19.1-29.6\end{array}$ & $\begin{array}{l}47.5(978) \\
37.9-57.3\end{array}$ & $\begin{array}{c}35.6(1494) \\
28.6-43.2\end{array}$ & $\begin{array}{c}20.47(409) \\
17.7-23.4\end{array}$ & $\begin{array}{l}27.4(509) \\
25.1-29.8\end{array}$ & $\begin{array}{l}23.9(918) \\
22.1-25.8\end{array}$ & $\begin{array}{c}55.5(1072) \\
47.4-63.2\end{array}$ & $\begin{array}{l}25.1(513) \\
16.1-36.8\end{array}$ & $\begin{array}{c}40.4(1585) \\
31.7-49.7\end{array}$ \\
\hline
\end{tabular}

Men's hot spot analysis showed that Ilam province $(P<.01)$ and Khuzestan $(P<.05)$ were hot spots for TPA volume (MET minutes per week) (Figure 1E). Ilam, Khuzestan, Kermanshah, and
Lorestan were hot spots for women, but the differences were not statistically significant than the national average $(P>.05)$

(Figure 1F). Table 3 depicts TPA, the average time spent at

Table 3 Total Physical Activity, the Average Time Spent at Work, Commute, Recreation, and Sedentary Behavior, Status by Gender in Iranian Adults Aged 15-64 years, 2007-2010

\begin{tabular}{|c|c|c|c|}
\hline \multirow[b]{2}{*}{ Province } & \multicolumn{3}{|c|}{ METs, min/wk } \\
\hline & Male, median (IQR) & Female, median (IQR) & Total, median (IQR) \\
\hline East Azerbaijan & $3960(9720)$ & $1080(3120)$ & $2100(5920)$ \\
\hline West Azerbaijan & $4040(9520)$ & $960(2720)$ & $1800(6040)$ \\
\hline Ardabil & $3420(8460)$ & $840(3540)$ & $1920(5880)$ \\
\hline Esfahan & $2880(8760)$ & $780(1920)$ & $1440(4680)$ \\
\hline Ilam & $5160(8000)$ & $2640(5360)$ & $3720(6960)$ \\
\hline Bushehr & $2720(6560)$ & $600(1760)$ & $1320(4000)$ \\
\hline Tehran & $2400(6240)$ & $960(2080)$ & $1440(3720)$ \\
\hline Chaharmahal and Bakhtiari & $6720(13,680)$ & $1560(4440)$ & $3200(8920)$ \\
\hline Razavi Khorasan & $4400(10,560)$ & $1440(3480)$ & $2400(6620)$ \\
\hline Khuzestan & $3600(8760)$ & $720(2040)$ & $1560(5180)$ \\
\hline Zanjan & $4800(10,720)$ & $1280(3240)$ & $2280(6260)$ \\
\hline Semnan & $3120(9680)$ & $840(1880)$ & $1440(4680)$ \\
\hline Sistan and Baluchestan & $2640(7200)$ & $480(1840)$ & $1140(4320)$ \\
\hline Fars & $2520(7000)$ & $720(2460)$ & $1440(4760)$ \\
\hline Qazvin & $3180(7440)$ & $840(2400)$ & $1680(4640)$ \\
\hline Qom & $2160(5280)$ & $660(1600)$ & $1200(3480)$ \\
\hline Kurdistan & $5280(13,200)$ & $1600(4320)$ & $2880(7800)$ \\
\hline Kerman & $4320(10,440)$ & $720(2760)$ & $1840(6400)$ \\
\hline Kermanshah & $3360(5280)$ & $1200(2600)$ & $1920(4080)$ \\
\hline Kohgiluyeh and Boyer-Ahmad & $5160(9080)$ & $2160(4640)$ & $3480(6560)$ \\
\hline Golestan & $4320(10,920)$ & $800(2880)$ & $1800(6360)$ \\
\hline Gilan & $4560(11,680)$ & $1080(3360)$ & $2280(6760)$ \\
\hline Lurestan & $7080(11760)$ & $1680(3480)$ & $3360(7920)$ \\
\hline Mazandaran & $5760(11,400)$ & $2040(4440)$ & $3360(7160)$ \\
\hline Markazi & $5520(11,940)$ & $1080(2880)$ & $2400(6720)$ \\
\hline Hormozgan & $2040(5760)$ & $560(1760)$ & $1040(3640)$ \\
\hline Hamedan & $4080(8280)$ & $840(2100)$ & $1800(4960)$ \\
\hline Yazd & $2880(9880)$ & $560(1860)$ & $1200(4800)$ \\
\hline South Khorasan & $4800(10,200)$ & $1680(4080)$ & $2680(6840)$ \\
\hline North Khorasan & $4560(10,080)$ & $960(3320)$ & $2160(6480)$ \\
\hline
\end{tabular}

Abbreviations: IQR, interquartile range; METs, metabolic equivalents. 
work, commute, recreation, and sedentary behavior, status by gender in Iranian adults aged 15-64 years, 2007-2010.

\section{Discussion}

The aim of this study was to examine the distribution of PA prevalence across different provinces of Iran using geographic maps of national surveys (2007-2010) to illustrate a geographic pattern of PA levels through GIS mapping. We used hot spot analysis (GetisOrd $\mathrm{Gi}^{*}$ ) to better understand that how different types of locations impact on prevalence of the PA in an objective way that does not rely on subjective judgments of the visual patterns.

Iran faces growing prevalence of low $\mathrm{PA}^{5}$; recent data suggest that the prevalence of low PA increased by $10 \%$ absolute points over 5 years, from $35 \%$ in 2006 to $45 \%$ in 2011.5

The descriptive findings of this study showed that Sistan and Baluchestan, Yazd, and Hormozgan that are located in the south and southeast of Iran consistently reported lower PA rates compared with other provinces. Although there is no clear explanation for this finding, a closer examination of PA pattern in these geographical units could shed light as to whether it is a chance finding or a systematic pattern. The objective hot spot analysis showed significantly low PA in the Kerman province; it is possible that the socioeconomic profile (low level of socioeconomic status) of this area explains this finding. ${ }^{27}$ This positive association between low socioeconomic status and overall PA level has been reported by other studies previously. ${ }^{26}$ Some other explanations for these findings relate to weather conditions in this region, which includes very high temperature and desert climate. Such conditions may discourage people to move without a vehicle while sports facilities (eg, sports fields and swimming pools) supply does not correspond with demand. Moreover, as few people farm or grow livestock and due to climatic conditions, activity may be reduced.

The other key finding is that geographical PA distribution has a particular pattern, as cold and mountainous areas such as north and northwest provinces, such as Ilam, Lorestan, and Mazandaran, mainly located along Alborz and Zagros Mountains range represent a higher level of PA. The hot spot analyses' findings illustrate Ilam as a hot region for high level of PA. A possible explanation for these results is that people in this area working with livestock and agriculture; the low prevalence of diabetes in this area supports this interpretation. ${ }^{25}$ Moreover, in mountainous areas in Iran, car use is infrequent, and incidental PA for transportation is bound to be higher. In the northern parts across the coastline, people may walk more and may be doing more swimming in the sea. Defining PA levels by geographic locations may produce a basis for supporting a better distribution of national resources for PA promotion as well as helping to shape effective health care.

Geographic information systems played a critical role in data analysis in our study by patterning the geographical variations in PA. If a higher resolution of participants' location such as town and small cities was available, more advanced spatial analysis such as smoothing or spatial regression analyses would be possible. However, the national survey data only provided a provincial level of living address; through this, GIS was used to display geographical variation at the PA levels. Although GIS enables to easily connect between health matters and geographic information, spatial analysis using GIS technologies assists investigating the association of PA and neighborhood, or local environment that can build a "PA-friendly" society including parks, recreational amenities, and road network such as a sidewalk, traces, and bike paths.
This GIS feature can be relevant to other health issues related to PA, such as obesity or chronic diseases. ${ }^{9,24}$

Physical inactivity is a serious public health issue in Iran, and measures to tackle it are needed. Despite the substantial progress made with developing national various PA policy documents in recent years, ${ }^{28,29}$ physical inactivity is still a low political priority in Iran, and there is not a national strategy. ${ }^{28}$ Without political commitment to a long-term national PA strategy covering both structured exercise and incidental PA (eg, active commuting), it is unlikely that the inactivity epidemic can be reversed. Increasing PA levels in the adult population will only be possible by developing and implementing a mixture of different interventions that are tailored to the geographical, climatic, and socioeconomic profile of a large county like Iran. Our data revealed consistent geographical patterns that can inform such interventions.

\section{Strengths and Limitations}

Strengths include the large nationally representative sample and use of the GPAQ, which allowed a comprehensive account of PA and the geographic analytical maps. To our knowledge, no such work has been previously conducted in Iran. Data were collected by trained professionals using consistent methods, and there was no need to employee retrospective harmonization. Our results must be interpreted in the context of the study limitations, which include the self-reported nature of the PA data that are subject to recall and social desirability bias.

\section{Conclusions}

This is the first detailed analysis of geographical patterning of PA prevalence in Iran using geographic maps. This study used GIS to serve in a visualization of PA status in different geographic locations; we identified large variation in PA across Iran provinces. The regions with low PA are predominately situated in the southeast and near to the center of Iran; conversely, high PA regions are mainly located in the west. These findings may enhance public awareness of the regional variations in PA and provide a basis for developing evidence-based health policies for vulnerable populations. In terms of linking PA data with regional-built environmental resources further utilizes that GIS should be implemented by future studies.

\section{Acknowledgments}

The authors thank Clinical Research Development Unit of Vasei Hospital, Sabzevar University of Medical Sciences for their assistance in this article. This research did not receive any specific grant from funding agencies in the public, commercial, or not-for-profit sectors. The authors declare that they have no conflict of interest. This study was approved by institutional review board of Tehran University of Medical Sciences with ethics committee code: IR.TUMS.SPH.REC.1395.514.

\section{References}

1. Braith RW, Stewart KJ. Resistance exercise training. Circulation. 2006;113(22):2642-2650. PubMed ID: 16754812 doi:10.1161/ CIRCULATIONAHA.105.584060

2. Haskell WL, Lee I-M, Pate RR, et al. Physical activity and public health: updated recommendation for adults from the American 
College of Sports Medicine and the American Heart Association. Circulation. 2007;116(9):1081-1093. PubMed ID: 17671237 doi:10. 1161/CIRCULATIONAHA.107.185649

3. Heath GW, Parra DC, Sarmiento OL, et al. Evidence-based intervention in physical activity: lessons from around the world. Lancet. 2012;380(9838):272-281. doi:10.1016/S0140-6736(12)60816-2

4. Foster C, Shilton T, Westerman L, et al. World Health Organisation to develop global action plan to promote physical activity: time for action. British J Sports Med. 2018;52:484-485.

5. Koohpayehzadeh J, Etemad K, Abbasi M, et al. Gender-specific changes in physical activity pattern in Iran: national surveillance of risk factors of non-communicable diseases (2007-2011). Int $J$ Public Health. 2014;59(2):231-241. PubMed ID: 24346180 doi:10. 1007/s00038-013-0529-3

6. Sahebkar M, Heidarian Miri H, Noormohammadpour P, et al. Prevalence and correlates of low physical activity in the Iranian population: national survey on non-communicable diseases in 2011. Scand J Med Sci Sports. 2018;28(8):1916-1924. PubMed ID: 29528518 doi:10. 1111/sms.13082

7. Fakhrzadeh H, Djalalinia S, Mirarefin M, et al. Prevalence of physical inactivity in Iran: a systematic review. J Cardiovasc Thorac Res. 2016;8(3):92-97. PubMed ID: 27777692 doi:10.15171/jcvtr.2016.20

8. World Health Organization. The global strategy on diet, physical activity and health (DPAS). 2005. http://www.who.int/nmh/wha/59/ dpas/en/. Accessed November 12, 2018.

9. Matthews SA, Moudon AV, Daniel M. Work group II: using geographic information systems for enhancing research relevant to policy on diet, physical activity, and weight. Am J Prev Med. 2009;36(4): S171-S176. doi:10.1016/j.amepre.2009.01.011

10. An R, Li X, Jiang N. Geographical variations in the environmental determinants of physical inactivity among US adults. Int J Environ Res Public Health. 2017;14(11):1326. doi:10.3390/ijerph14111326

11. Schuurman N, Peters PA, Oliver LN. Are obesity and physical activity clustered? A spatial analysis linked to residential density. Obesity. 2009;17(12):2202-2209. PubMed ID: 19390521 doi:10. 1038/oby.2009.119

12. Rogerson PA. Statistical methods for the detection of spatial clustering in case-control data. Stat Med. 2006;25(5):811-823. PubMed ID: 16453374 doi:10.1002/sim.2426

13. Yeom H-A, Jung D, Choi M. Adherence to physical activity among older adults using a geographic information system: Korean national health and nutrition examinations survey IV. Asian Nurs Res. 2011; 5(2):118-127. doi:10.1016/S1976-1317(11)60020-0

14. An R, Xiang X, Yang Y, Yan H. Mapping the prevalence of physical inactivity in US states, 1984-2015. PLoS ONE. 2016;11(12): e0168175. PubMed ID: 27959906 doi:10.1371/journal.pone.0168175

15. Holakouie-Naieni K, Mostafavi E, Boloorani AD, Mohebali M, Pakzad R. Spatial modeling of cutaneous leishmaniasis in Iran from 1983 to 2013. Acta Trop. 2017;166:67-73. PubMed ID: 27836499 doi:10.1016/j.actatropica.2016.11.004

16. Esteghamati A, Khalilzadeh O, Rashidi A, et al. Association between physical activity and insulin resistance in Iranian adults: National Surveillance of Risk Factors of Non-Communicable Diseases
(SuRFNCD-2007). Prev Med. 2009;49(5):402-406. PubMed ID: 19744508 doi:10.1016/j.ypmed.2009.09.005

17. Esteghamati A, Khalilzadeh O, Rashidi A, Kamgar M, Meysamie A, Abbasi M. Physical activity in Iran: results of the third national surveillance of risk factors of non-communicable diseases (SuRFNCD-2007). J Phys Act Health. 2011;8(1):27-35. PubMed ID: 21297182 doi:10.1123/jpah.8.1.27

18. Noormohammadpour P, Mansournia MA, Koohpayehzadeh J, et al. Prevalence of chronic neck pain, low back pain, and knee pain and their related factors in community-dwelling adults in Iran: a population-based national study. Clin J Pain. 2017;33(2):181-187. PubMed ID: 27258995 doi:10.1097/AJP.0000000000000396

19. World Health Organization. Global physical activity questionnaire (GPAQ) analysis guide. 2012. https://www.who.int/ncds/surveillance/ steps/GPAQ\%20Instrument\%20and\%20Analysis\%20Guide\%20v2. pdf. Accessed November 12, 2018.

20. Bull FC, Maslin TS, Armstrong T. Global physical activity questionnaire (GPAQ): nine country reliability and validity study. J Phys Act Health. 2009;6(6):790-804. PubMed ID: 20101923 doi:10.1123/ jpah.6.6.790

21. Ainsworth BE, Haskell WL, Whitt MC, et al. Compendium of physical activities: an update of activity codes and MET intensities. Med Sci Sports Exerc. 2000;32(9)(suppl):S498-S504. PubMed ID: 11224794 doi:10.1097/00005768-200009001-00009

22. World Health Organization. WHO STEPS surveillance manual: the WHO STEPwise approach to chronic disease risk factor surveillance / Noncommunicable Diseases and Mental Health. 2005. http://apps. who.int/iris/handle/10665/43376. Accessed November 12, 2018.

23. Mansournia MA, Altman DG. Inverse probability weighting. $B M J$. 2016;352:i189. PubMed ID: 26773001 doi:10.1136/bmj.i189

24. Brownson RC, Hoehner CM, Day K, Forsyth A, Sallis JF. Measuring the built environment for physical activity: state of the science. Am J Prev Med. 2009;36(4):S99-S123.e112. doi:10.1016/j.amepre.2009. 01.005

25. Haghdoost A, Rezazadeh Kermani M, Sadghirad B, Baradaran H. Prevalence of type 2 diabetes in the Islamic Republic of Iran: systematic review and meta-analysis. East Mediterr Health J. 2009;15(3):591-599. PubMed ID: 19731775 doi:10.26719/2009. 15.3.591

26. Juneau C, Benmarhnia T, Poulin A, Côté S, Potvin L. Socioeconomic position during childhood and physical activity during adulthood: a systematic review. Int J Public Health. 2015;60(7):799-813. PubMed ID: 26298440 doi:10.1007/s00038-015-0710-y

27. Statistical Research and Training Center. Annual report. 2015. http:// srtc.ac.ir/Portals/0/PropertyAgent/578/Files/38/Annual\%20Report\% 201394.pdf. Accessed November 12, 2018.

28. Mounesan L, Sepidarkish M, Hosseini H, et al. Policy brief for promoting physical activity among Iranian adolescents. J Isfahan Med Sch. 2013;31(233):510-520.

29. Daugbjerg SB, Kahlmeier S, Racioppi F, et al. Promotion of physical activity in the European region: content analysis of 27 national policy documents. J Phys Act Health. 2009;6(6):805-817. PubMed ID: 20101924 doi:10.1123/jpah.6.6.805 